\title{
Y a-t-il un chamane dans le cockpit ? Sur quelques travaux d'histoire et de sociologie de l'aéronautique
}

\section{Pierre Lemonnier}

\author{
(2) OpenEdition \\ Journals \\ Édition électronique \\ URL : https://journals.openedition.org/tc/130 \\ DOI : $10.4000 /$ tc. 130 \\ ISSN : 1952-420X \\ Éditeur \\ Éditions de l'EHESS
}

\section{Édition imprimée}

Date de publication : 1 avril 2004

Pagination : 141-164

ISSN : 0248-6016

\section{Référence électronique}

Pierre Lemonnier, «Y a-t-il un chamane dans le cockpit ? Sur quelques travaux d'histoire et de sociologie de l'aéronautique », Techniques \& Culture [En ligne], 42 | 2004, mis en ligne le 06 novembre 2007, consulté le 29 septembre 2022. URL : http://journals.openedition.org/tc/130 ; DOI : https:// doi.org/10.4000/tc. 130

Ce document a été généré automatiquement le 29 septembre 2022.

Tous droits réservés 


\title{
Y a-t-il un chamane dans le cockpit? Sur quelques travaux d'histoire et de sociologie de l'aéronautique
}

\author{
Pierre Lemonnier
}

Physiquement, un avion est un objet qui se meut dans les airs en emportant (généralement), outre la charge qu'il déplace, celui ou celle qui en dirige les évolutions. C'est aussi un sujet d'étude de choix pour qui se propose de rendre compte de la diversité des cultures et des sociétés en choisissant l'entrée "action sur la matière ", car on peut soulever à propos de ce produit-clef de notre monde industriel toutes sortes de questions, que son développement récent permet de surcroît d'étendre dans le temps, jusqu'à son origine même, il y un peu plus d'un siècle. La problématique de l'ethnologie des techniques s'y applique et n'a rien de trivial : pourquoi observe-t-on cet appareil-là, ici et maintenant? Pourquoi cette manière de le faire voler ou de l'entretenir? En quoi cette machine et sa mise en œuvre sont-ils propres (ou non) aux gens qui l'ont fabriquée ou la pilotent ? Dans bien des cas, il serait possible de décrire et comprendre les actions physiques par lesquelles on fabrique, fait voler ou prend soin d'un avion, d'observer des variantes au sein de ces pratiques et d'expliquer les choix dont elles résultent. Par exemple, je n'ai pas besoin d'être moi-même pilote ou mécanicien pour réaliser que les violentes glissades en « $\mathrm{S}$ " imposées au petit monomoteur qui me transporte chez les Anga vont le freiner sur l'air " par opposition de fuselage » et remplacent, in extremis, l'action de volets dont la panne a entraîné cette réaction particulière du pilote de la "Missionary Aviation Fellowship $»^{1}$. Et la manière dont celui-ci vérifie, pendant de longues secondes, le serrage de chacun des écrous que son collègue vient pourtant de bloquer sous ses yeux révèle la minutie avec laquelle ces pères de famille protestants entretiennent les Cessna qu'ils font voler au-dessus des forêts, des brumes et des montagnes de Nouvelle-Guinée; y compris lors d'un banal changement de courroie d'alternateur (ou était-ce une magnéto ?) au beau milieu d'un 
vol charter emmenant un ethnologue vers les chamanes et les rituels qui occupent sa vie professionnelle.

Qu'il s'agisse de manier une clef dynamométrique ou de jouer des pieds sur un palonnier, le lien entre le geste humain que j'observe et son effet matériel m'est compréhensible. Mon pilote tourne son volant à gauche et pousse le pied droit, et, quelques mètres de tringles et de câbles plus loin, des gouvernes et des ailerons s'orientent de manière à nous faire voler en crabe et perdre de la vitesse. Mais ce Cessna « 206 Turbostationair» est un avion dont la conception remonte à une quarantaine d'années (1955 pour son ancêtre, le « $172 »)$, pendant lesquelles beaucoup de choses ont changé.

3 Les «actions sur la matière " effectuées à chaque instant de la fabrication ou de l'utilisation d'un aéroplane moderne sont si complexes que les méthodes de la technologie culturelle atteignent peut-être leurs limites. En particulier, dès qu'il s'agit d'avions de ligne conçus ces vingt dernières années (ou d'appareils militaires), le subtil entrelacs de représentations qui inscrit une tâche technique dans l'ensemble d'une culture devient difficilement lisible dans des chaînes opératoires. Que nous disent, à propos des rapports entre techniques et société, cette façon de rectifier des aubes de turbines usagées, de superposer à la main des nappes de matériaux composites, ou cette pression de l'index par laquelle un pilote enclenche ou supprime l'une des dizaines de procédures automatiques qui l'aident à conduire sa machine? À l'ethnologue ordinaire, pas grand-chose, car indépendamment des facteurs économiques ou politiques qui pèsent également sur les choix techniques (la décision de réaliser Concorde, de créer Airbus ou d'autoriser le Boeing 777 à voler à une distance toujours plus grande des terrains de secours qui l'accueilleraient en cas d'arrêt malvenu de l'un de ses deux monstrueux réacteurs), produire un avion est une affaire si hautement spécialisée qu'un ingénieur peut y perdre son latin. Non seulement les fabrications des cellules, des moteurs, des freins, des trains ou de l'avionique n'ont guère de points communs entre elles (hormis lors de l'assemblage final!), mais au sein d'une même industrie, disons celle des moteurs, les domaines sont légion (mécanique des fluides, résistance des matériaux, systèmes de mesure, gestion informatisée, etc.). Quant au pilotage, il s'est profondément modifié avec l'installation d'ordinateurs dans les appareils. Les câbles et les poulies ne sont plus là que pour suppléer à une improbable (et cauchemardesque) panne électronique, et un «mini-manche » envoie des impulsions électriques à des moteurs qui entraînent les surfaces mobiles.

Ici, faire de la technologie culturelle ou écrire cette "histoire technique des techniques » que réclamait Lucien Febvre n'est pas une mince affaire et demande que le chercheur soit aussi un ingénieur spécialisé dans la branche dont il traite. Ceux-ci ne manquent pas et des centaines de livres et plusieurs revues de très haute qualité (comme Air \& Space/Smithsonian aux USA, Aeroplane Monthly et FlyPast au Royaume Uni, ou Le Fana de l'Aviation en France) rapportent par le menu le développement ou la « carrière » d'avions les plus divers (Crouch, Jakab 1994, Hooven 1978 ou Owen 1982 parmi des centaines d'exemples). Mais, bien que toujours remarquablement documentés, ces travaux sont essentiellement descriptifs. Les ouvrages de synthèse sont rares - par exemple Vincenti (1994) pour les trains d'atterrissage, Bodemer et Laugier (1979) ou Nahum (1987) pour les moteurs-, et les études de technologie comparée encore plus (Quilici 2000). Sauf exception (Chadeau 1991, Quilici 1993, Vincenti 1990), les sciences humaines -j'entends la prise en compte de pratiques et de 
représentations diverses dans l'élaboration et la réalisation d'un avion- sont absentes de ces milliers de pages.

5 L'histoire de l'aviation signale amplement l'existence d'options plus ou moins arbitraires, d'effets de mode ou de comportements routiniers dans lesquels l'efficacité n'a guère sa place (Lemonnier 1996), mais à propos de ces manières de fabriquer un appareil, plutôt que de décrire et d'expliquer le choix de techniques ou de matériaux particuliers, les historiens de l'aéronautique parlent du contexte économique et politique de l'industrie aéronautique (par exemple Bénichou 2000, Chadeau 1987, Edgerton 1991, Sparaco 2002), et, plus encore, de la saga d'un constructeur ou d'un modèle particulier.

6 C'est dire l'importance d'ouvrages comme les deux livres que J.-Cl. Fayer vient de consacrer à l'histoire du Centre d'Essais en Vol entre 1945 et $1960^{2}$. Vol d'essais rapporte la constitution puis les premières années d'activité du CEV, à Brétigny, Istres et Cazaux. Héritier du Centre d'essais des matériels aériens (CEMA) installé à Villacoublay avant la guerre, mondialement renommé pour ses méthodes d'essai et son matériel d'enregistrement des mesures effectuées en vol, dont le "hussenographe » (Gaillard 1954: 55), le CEV fut chargé d'évaluer les prototypes présentés par plusieurs constructeurs en réponse à chaque "programme » (définition d'un type d'avion et de ses performances minimum) de l'État ou de l'OTAN. Au-delà de sa responsabilité dans l'orientation des choix et des commandes ministériels, le CEV joua de fait « un rôle d'arbitre et de conseiller entre les industriels fournisseurs et les utilisateurs militaires ou civils » (Fayer I : 7). En même temps que la mise au point de matériels nouveaux des avions, mais aussi des sièges éjectables, des systèmes d'alimentation en oxygène, des combinaisons " anti-G ", des parachutes, des procédés de télécommunication ou de ravitaillement en vol, etc.-, les dirigeants et le personnel du CEV eurent à inventer une réglementation et des procédures d'essais à une époque qui fut l'une des plus innovantes de l'histoire de l'aéronautique. Une vingtaine d'avions voyaient le jour chaque année, dont les premiers chasseurs à réaction, les prestigieuses machines qui s'attaquèrent peu à peu aux vitesses supersoniques (S.0. 6000 "Triton", "Leduc", " Grognard », « Espadon ", " Gerfaut », " Griffon ", « Trident »), et tous les prototypes dont les appareils militaires devaient dériver pendant cinquante ans ("Ouragan», " Mystère ", "Vautour ", "Étendard ", " Mirage »). Ce fut aussi l'époque de la percée des techniques françaises en matière d'hélicoptères (« Alouette») ou d'avions de ligne ("Caravelle »). Celle des records, aussi.

7 L'ouvrage décrit de manière très détaillée la vie du centre année par année : les types d'essais menés, le personnel en place, les avions testés, les drames fréquents. L'auteur estime modestement avoir écrit un ouvrage « exclusivement factuel », mais sa minutie, les extraits de rapports d'essais et les dizaines d'« encadrés » dans lesquels d'anciens pilotes ou ingénieurs racontent des épisodes particuliers de leur vie au CEV ou évoquent celle d'un camarade disparu en vol en disent long sur cette recherche de solutions techniques "par tâtonnements » qui demandait parfois plusieurs dizaines de vol pour un unique problème (Fayer I : 11). Il n'y a pas là d'enquête sociologique, mais les témoignages rassemblés donne une image d'ensemble de la vie, des motivations et du rôle irremplaçable des navigants d'essai.

8 Consacré à tous les prototypes français de cette période, le second livre de J.-Cl. Fayer (Prototypes de l'aviation française, 1945-1960) est constitué de fiches synthétiques illustrées par des photographies faites à l'époque au CEV. Elles indiquent les performances de 
chaque appareil et, surtout, l'avis porté sur son « comportement en vol» et le sort qui fut le sien. Il offre également une annexe sur les «Buts et méthodologies des évaluations officielles » qui décrit avec la plus grande clarté les procédures employées et leurs raisons d'être (Fayer II : 309-311).

9 Complémentaires l'un de l'autre et d'utilisation aisée, ces deux livres constituent des ouvrages de référence pour l'étude de l'histoire de l'aviation française de l'aprèsguerre. À défaut d'une enquête historique sur le monde des concepteurs, qui reste à faire, ils offrent un inestimable regard sur un acteur-clef des choix aéronautiques -du fait des orientations que les navigants d'essais pouvaient alors suggérer (ou exiger), mais également pour leur rôle dans l'acceptation ou le rejet de formules techniques qui ont fixé pour des décennies le cadre général des innovations.

10 À défaut de s'intéresser à l'histoire de l'aéronautique, la sociologie de l'innovation s'intéresse à l'aéronautique contemporaine ${ }^{3}$. Ou, plutôt, à certains aspects du monde de l'aviation. Pour quelque raison, les spécialistes des sciences humaines ne s'aventurent pas dans des domaines relevant de la mécanique. Tout laisse penser que les choix dont résultent une voilure (celle, partiellement en "composites ", de l'Airbus A-380 ou du prochain Boeing 7E7), des hélices rapides (celles du futur avion de transport militaire européen), l'éventuelle activation électrique (et non plus hydraulique) des freins, ou le développement de biréacteurs de plus en plus puissants (tel le Boeing 777), résultent autant de décisions sociologiquement ou politiquement complexes et de controverses que n'importe quelle autre technique moderne ${ }^{4}$. Cependant, sauf erreur, ces affaires de mécanique échappent aux analyses de sciences humaines. Plusieurs études récentes ont en revanche mis l'accent sur le pilotage, singulièrement sur la manière dont l'automatisation des avions soulève des questions allant bien au-delà de la simple conduite des appareils.

11 Mais surtout, trois types d'« intelligences » dirigent désormais les avions : celle des pilotes, celle des ingénieurs qui ont programmé les ordinateurs embarqués avec lesquels le pilote et le co-pilote dialoguent du bout des doigts, et celle des contrôleurs aériens, qui, du sol, leur indiquent la route à suivre. Le métier des pilotes s'en trouve transformé, mais ce n'est là qu'un aspect des évolutions contemporaines de l'aéronautique civile. Cette "avionique" dont la maîtrise bouleverse leur travail représente une part croissante du prix d'un avion, et développer et tester des "systèmes » est semble-t-il devenu au moins aussi important que mettre au point de nouveaux matériaux ou techniques de construction. Dans la compréhension de ce processus d'innovation continu, le pilotage est bien davantage qu'un point de départ commode pour rendre compte de l'usage de machineries, de systèmes informatiques ou de mode de conduite des avions en constante mutation : tant en amont qu'en aval des nouveautés techniques, les pilotes sont des acteurs centraux du processus lui-même. C'est à eux que sont consacrés deux livres récents de sociologie des techniques ${ }^{5}$.

Écrit par Caroline Moricot, Des avions et des hommes traite de l'introduction massive d'automatismes gérés par ordinateur dans le pilotage des avions de ligne, à la fin des années 1980, notamment à bord de l'Airbus A-320, dont le lancement fut marqué par des controverses (à propos de la suppression du mécanicien navigant, qui s'accéléra à cette occasion, et à la suite de deux catastrophes, à Absheim et au Mont Sainte Odile). 
Avec l'arrivée de ces avions à « cockpit de verre » (glass cockpit), les tâches des pilotes furent modifiées et il y avait alors lieu de chercher à comprendre comment ils ont réagi -comment ils ont utilisé ces avions nouveaux-, et de replacer cette " appropriation » d'une "nouvelle génération " d'appareils dans le cadre d'une plus large réflexion sur les rapports entre les hommes et les systèmes automatisés, qui soulève ici de surcroît la question de la gestion de la sécurité dans le monde industriel moderne.

Pour ce faire, plutôt que d'étudier l'innovation du côté des concepteurs ou d'analyser les circonstances ayant mené à un accident, l'auteur a choisi de se tourner vers l'usage ordinaire des avions en ligne, lorsqu' «il ne se passe "rien" » (CM : 9). Son travail est fondé sur le rapprochement d'entretiens (une centaine d'heures d'interviews) et d'observations directes (une quinzaine de « rotations » vécues à côté des pilotes depuis l'intérieur du cockpit), avec le dépouillement de près de 800 questionnaires remplis par des commandants de bord et des copilotes. Cette démarche " anthropo-sociologique " convaincante aboutit à un ouvrage passionnant, facile à lire et mené de main de maître, qui donne une description claire de ce qui s'est passé sur les avions au moment où les équipages se sont " approprié » les glass-cockpits qu'on leur proposait : en établissant de nouveaux liens avec eux, c'est-à-dire en « reconstruisant une représentation de l'objet technique, de leur travail, d'eux-mêmes » (CM : 7).

Couplés à des écrans cathodiques en couleur, qui remplaçaient la kyrielle de cadrans précédemment utilisés, une multitude de calculateurs s'intercalent désormais entre le pilote et la machine. Au lieu d'agir plus ou moins directement sur des organes mécaniques, les pilotes de ligne " pianotent » sur des claviers et « dialoguent » avec des ordinateurs pour «entrer » des données concernant le vol ou choisir des procédures d'évolution (montée, virage, désignation d'une direction, etc.). Par la mise en relation de différents calculateurs, correspondant à autant de fonctions du pilotage ou d'organes de l'avion, le Flight Management System se comporte comme un super-pilote automatique. Si les pilotes sont satisfaits de déléguer à des ordinateurs les opérations mentales complexes nécessaires à la navigation, en revanche, ils n'apprécient guère que le "système " ne transmette leurs ordres à l'avion qu'après en avoir apprécié l'adéquation, par exemple lorsqu'un dispositif dit de "protection du domaine de vol » s'oppose à toute action entrainnant une évolution ou une attitude dangereuse pour la machine. Nombreux sont ceux qui ne comprennent pas la logique ou les réactions du système. Non seulement ils s'inquiètent «d'automatismes qui font leurs trucs dans leur coin ", mais ils savent qu'il ne leur est plus possible de tout comprendre.

Cette incertitude s'est trouvée augmentée par la disparition de certaines sensations dans les glass-cockpits, notamment en raison de l'absence de "retour d'information » sur les effets matériels de leurs gestes: au volant (ou "manche ») indirectement relié aux gouvernes s'est substitué une sorte de joystick (dit «mini-manche ») dans lequel, explique un pilote, "on ne sent pas l'effort, on ne sent pas de résistance » (CM : 253), alors qu'« un avion normal, il faut le piloter tout le temps, on ne peut pas lâcher le manche »; quant aux manettes des gaz qui, naguère, se déplaçaient seules au gré du pilote automatique, permettant ainsi à l'équipage de jauger le régime des moteurs sans regarder un seul cadran, en repérant leur position d'un geste de la main, elles restent désormais immobiles. La suppression des mécaniciens navigants et de la «culture mécanique » qu'ils détenaient n'arrange évidemment rien. Au bout du compte, le pilote " a perdu les moyens de se forger une représentation acceptable (pour lui) des différents systèmes, de leur fonctionnement et de leurs interactions pour une raison 
très simple qui est que, pour une part, ces systèmes sont devenus immatériels !» (CM : 202). Cette "rupture du cadre de compréhension » ( $C M: 75)$ génère des incertitudes d'autant plus pesantes qu'en dernier ressort ce sont les pilotes qui interprètent les informations éventuellement fausses que leur relaient les écrans cathodiques, et ce sont toujours eux qui portent la responsabilité de la conduite de l'appareil.

L'« appropriation» de ces cockpits nouveaux a consisté pour les hommes de l'art à développer des connaissances et des pratiques nouvelles -et non prévues par les concepteurs des avions - afin de rendre plus familiers les systèmes qu'ils contiennent. D'abord, au fur et à mesure qu'ils se communiquaient les uns aux autres les réactions surprenantes ou les pannes des "systèmes", les pilotes ont repris confiance: en découvrant le répertoire des "vraies-fausses pannes" à ignorer, mais aussi par de nouveaux gestes. Face à une information douteuse ou inintelligible, par exemple, on décide de "tirer le breaker", de couper l'alimentation du système en cause, ou on appuie la touche "reset» qui le "décoince ", c'est-à-dire qu'on substitue une «représentation mécanique " à l'insondable immatérialité qui se cache derrière les écrans. Mais aussi, et au grand dam des concepteurs, en repassant en pilotage manuel dans des circonstances qui permettent aux équipages de mettre en avant ce par quoi ils caractérisent leur métier, c'est-à-dire leur habileté, leur "sens de l'air » et leur responsabilité : en déconnectant les automates et en assurant manuellement le pilotage chaque fois qu'ils doutent des systèmes; et en prenant régulièrement eux-mêmes les commandes de l'appareil à un moment ou l'autre du vol «pour maintenir leur habileté » (CM : 252) et... pour se faire plaisir.

17 Constatant à son tour que les objets n'existent que par le sens qu'on leur donne, ce dont nul ethnologue des techniques ne saurait douter -dans le livre de C. Moricot, l'honneur de porter les couleurs de la technologie culturelle revient au regretté A.-G. Haudricourt (CM : 222)-, l'auteur souligne à juste titre que ce sont les imperfections des avions, ainsi que le bricolage ou l'inventivité par lesquels les pilotes y ont répondu, qui ont permis leur appropriation : " [...] le processus d'appropriation se niche dans les espaces "imparfaits" des systèmes " (CM : 271), dans cette "marge d'indétermination » dont l'auteur reprend l'idée à G. Simondon. C'est pour répondre aux pannes, vraies ou fausses, et pour rendre plus confortable cet «espace d'incertitude » que sont devenus les cockpits envahis par les ordinateurs, que les pilotes ont réaffirmé leur expérience collective et revendiqué l'image qu'ils ont d'eux-mêmes : celle de gens qui ont le «sens de l'air ", possèdent l'habileté de faire évoluer un avion et portent la responsabilité ultime de l'appareil; mais aussi celle d'un collectif se reconnaissant un système de valeur centré sur la sécurité. Bref, l'adaptation des pilotes aux imperfections des systèmes leur a permis « de conserver leur qualification et leur autonomie » (VS : 22).

Deux autres résultats dont la portée dépasse l'aéronautique se dégagent de ce beau livre. D'abord, «l'intensification des automatismes dans les glass-cockpits rend la présence des hommes encore plus nécessaire» $(\mathrm{CM}: 12,362)$ : «il leur revient de décider en permanence de la fiabilité des informations affichées sur les écrans ». Cette constatation devrait logiquement conduire les concepteurs à se préoccuper davantage de l'avis et des pratiques de ceux dont on veut minimiser l'intervention dans la conduite des avions, par exemple en interdisant purement et simplement l'atterrissage manuel en " catégorie deux » (visibilité réduite) $(\mathrm{CM}: 314,325)$. Les pilotes considèrent que «le progrès n'est pas nécessairement synonyme de plus d'automatisation » (CM : 351) -et l'avenir leur a déjà donné partiellement raison: d'un côté, même si le 
brouillard ou la nuit bloquent entièrement la vue d'un pilote, il lui est aujourd'hui possible de se poser " manuellement " grâce aux révolutionnaires Head Up Displays qui projettent devant leurs yeux une image virtuelle ou infrarouge extraordinairement précise $^{6}$; de l'autre, on expérimente des systèmes associant un GPS aux automates de l'avion qui permettent aujourd'hui à un énorme Boeing 747 de se poser «au mètre près ", dit-on, sans intervention humaine... La phase d'appropriation des nouveaux appareils/systèmes devrait être prise en compte lors de leur conception même (CM : 68), ce qui ne fut apparemment pas le cas au moment de la définition d'un appareil comme l'A-320. Un pilote déclare même -ironiquement, on l'espère : "Je crois que dans l'équipe d'étude, il n'y avait pas de pilote » (CM : 323).

Le pilote et l'ingénieur, écrit Moricot, "projettent dans la "machine" leur représentation $d u$ vol, leur imaginaire de la technique et du progrès » (CM : 321) : "pour le pilote, le danger, c'est l'intégration trop poussée où la machine, rendue autonome, lui échappe, s'emballe et devient incohérente ; pour le concepteur (tel que l'imagine le pilote), c'est l'homme et le potentiel d'erreur qu'il porte en lui, il n'est pas fiable » (CM : 324). Rappelant que le sociologue (en l'occurrence V. Scardigli) met bel et bien au jour chez ces ingénieurs ces représentations que leur prêtent les usagers des cockpits, l'auteur plaide pour un rapprochement des deux communautés pendant la conception des appareils ( $\mathrm{CM}: 148)$. Les pilotes de ligne regrettent amèrement ce manque de dialogue entre eux et les constructeurs, tout en contestant la place que les pilotes d'essai y tiennent déjà (CM : 334-336).

Comme on le voit, la question de la part respective à attribuer aux pilotes et aux automatismes dans la conduite des avions tourne autour de la confiance que l'on fait aux uns et aux autres en vue de l'obtention d'une sécurité aérienne maximum. Ici, l'opposition entre les opinions respectives des pilotes de ligne et les constructeurs est totale : « Ce que les pilotes traduisent [...] par "difficulté dans la communication avec la machine", les constructeurs le traduisent par "problème imputable à l'équipage" et la communauté aéronautique par "facteur humain" ( $\mathrm{CM}: 148)$. C. Moricot ne tranche pas, mais, à la lire, le lecteur a néanmoins l'impression que les automates et ceux qui les conçoivent ou les imposent ont ici de lourdes responsabilités : «la plupart du temps, le facteur humain semble un coupable tout désigné alors que peut-être, comme le suggère Véronique de Keyser", "l'erreur humaine" n'est qu'un symptôme des carences du système » (CM : 90). Parfois, les concepteurs font des erreurs modérées. Sur l'A-320 (qui n'est pas le seul dans ce cas), le constructeur a ainsi cru bon d'installer les phares sur le train d'atterrissage, ce qui empêche le pilote de signaler sa position à un autre appareil par un appel de phares ou de mesurer l'intensité du givre ou des précipitations dans un nuage. Mais, parfois, la "carence du système » a des conséquences dramatiques: un pilote parle d'un « changement intempestif de mode de guidage vertical, non détecté par l'équipage, ayant conduit celui-ci à demander par ses gestes un taux de descente cinq fois plus rapide que le maximum souhaité », scénario dont C. Moricot (p. 192) note qu'il serait du type observé lors de la catastrophe de Strasbourg (Mont Sainte Odile). Dans un tel cas, le pilote s'est trompé dans ses gestes, mais ce sont les systèmes qui l'ont « surpris ».

21 À ce propos, il y aurait sans doute lieu de mesurer le fossé qui semble exister entre les déclarations des pilotes de ligne (ou les observations faites dans un poste de pilotage un jour où il ne se passe "rien») et cet autre monde, tout aussi réel, qu'est celui des cockpits dans lesquels les pilotes ne se montrent pas respectueux de ces « règles de 
l'art " dont l'oubli produit des accidents et conduit à un "scepticisme fondamental » qui invite à être toujours méfiant (CM : 162, 164). Si j'ai évoqué plus haut mes propres «observations» en Papouasie Nouvelle-Guinée, ce n'est pas pour m'inventer une compétence que je n'ai pas, mais parce que ce que j'y ai observé malgré moi à bord de petits avions (et d'hélicoptères) de compagnies dites de «troisième niveau » n'était pas toujours en accord avec les pratiques dont il est question ici. D'un côté, dans ce petit pays, l'obsession de la sécurité est la même qu'ailleurs et ses règles sont respectées : même "au milieu de nulle part ", un avion ne décolle pas sans autorisation, ni sans avoir indiqué sa destination et son horaire approximatif d'arrivée à un contrôleur aérien. Une fois en vol, le pilote ne cesse de renseigner les autres appareils et ce contrôleur sur sa route, la météo rencontrée, ou la position d'un avion entrevu ici ou là, ce qui est une façon de suppléer l'absence de radar dans ce pays où l'on vole à vue en modifiant sans cesse son plan de vol, au gré des nuages et de la pluie tropicale qui «bouchent » une vallée ou un col en quelques minutes. Ici comme ailleurs, on vérifie plusieurs fois dans une journée que l'essence d'un réservoir ne contient pas d'eau, on attend le feu vert de la tour de contrôle avant de décoller parce qu'une vague nappe de brouillard pourrait gêner un éventuel retour vers le terrain en cas de problème au décollage, et on cloue au sol une machine dans l'huile de laquelle on a trouvé un minuscule morceau de limaille. Mais, à côté de pilotes qui se comportent comme la communauté aéronautique exige qu'on le fasse, il en est d'autres qui prennent quelque liberté avec les « règles de l'art » : qui démarrent un Cessna avec autant d'attention que je mets ma voiture en route, sans effectuer aucune vérification ni enfiler les bretelles de leur harnais de sécurité ; passent systématiquement au ras des arbres, "pour rire ", et ne s'alignent qu'au dernier moment sur l'axe de la piste où ils atterrissent; décollent sous la pluie battante, avec trente mètres de visibilité d'un terrain de brousse littéralement enserré par des collines ; « font la course » pour se poser les premiers afin de proposer leurs services à d'éventuels passagers, etc.

On pourrait en déduire que les bush pilots de Papouasie n'auraient pas leur place à " Air France » ou chez «Qantas ». Ou que la Papouasie se contente d'une sécurité au rabais ${ }^{8}$. Pour ma part, je suis au contraire frappé par la ressemblance (partielle, heureusement pour moi!) entre les observations involontaires que j'ai faites dans ce pays depuis vingt-cinq ans et ce que je lis régulièrement dans Flying - probablement la plus importante et la meilleure revue aéronautique au monde-, où la question du "facteur humain " est abordée d'une manière qui mérite peut-être d'être prise en considération dans une optique comparative.

Des impressions de lecture ne valent pas des statistiques, mais cette revue américaine donne de certains pilotes professionnels une image encore plus négative que celle $\mathrm{du}$ " pilote moyen » à la place duquel tentent de se mettre les pilotes d'essai dont il sera question un peu plus loin. Page après page, elle martèle, littéralement, des consignes de sécurité. La rubrique «I learned about flying from that " (759 historiettes à ce jour) est écrite par des pilotes qui ont frôlé la catastrophe pour avoir oublié ou sous-estimé l'une de ces règles. Quant à la chronique "On The Record», elle est constituée de résumés d'accidents ou d'incidents survenus dans l'aviation générale qui, presque sans exception, soulignent que « le pilote a omis de... », « n'a pas réussi à » faire ceci ou cela ; « n’a pas fait répéter le contrôleur...»; «a donné une fausse position... »; « n’a tenu aucun compte » des renseignements météorologiques qu'on lui fournissait; etc. Pour 
Flying, qui est une revue écrite par des pilotes, majoritairement des pilotes de ligne, l'accident vient clairement de celui qui est aux commandes.

La même impression se dégage de la rubrique «Aftermath » (« Suites [d'un accident]) », qui analyse les rapports d'enquête publiés par le National Safety Board américain concernant des accidents survenus à des appareils de transport. On y trouve certes l'exposé de situations rappelant très précisément celles dont parle $\mathrm{C}$. Moricot, où des pilotes d'Airbus A-300 ne réalisent pas qu'ils luttent sans espoir (et avec de dramatiques conséquences) contre un pilote automatique pourtant enclenché par eux, mais inventé par des ingénieurs n'ayant pas imaginé la confusion que l'automate peut créer chez un équipage (Garrison 1998). Mais ces rapports d'accidents soulignent le plus souvent des défaillances humaines. Les pilotes professionnels ne sont pas seuls en cause -les contrôleurs et les avions mal entretenus ont leur part de faiblesse dans ces drames-, mais leur manque de vigilance, la liberté qu'ils prennent avec les procédures, ou l'àpeu-près dont ils se contentent fait frémir : tel pilote qui n'avait bouclé ni ceinture ni harnais s'accroche à son volant lorsque l'appareil rebondit durement sur la piste, si bien que celui-ci fait la roue et explose (Garrison 1985); un autre est si dangereux que personne ne veut l'avoir pour co-pilote (Garrison 1994); d'autres encore font malencontreusement confiance à leur mémoire plutôt qu'à la check-list, négligent de redoubler de prudence lors du délicat passage du vol aux instruments au vol à vue en phase d'atterrissage, ou chargent leur appareil sans tenir compte de la position du centre de gravité (Garrison 1999a, 2003, 1999b). Il est possible que Flying choisisse d'analyser des accidents impliquant la responsabilité d'équipages qui n'ont pas respecté les consignes de sécurité pour rendre encore plus plausible son credo sécuritaire, ou que ce magazine soit rédigé par une bande de suppôts des constructeurs. Mais ces cas qui se comptent par dizaines confortent plutôt l'idée commune selon laquelle $70 \%$ des accidents sont dus à un facteur humain (CM : 92). Bref, pour le lecteur de Flying, les pilotes professionnels semblent moins parfaits que ceux interviewés par C. Moricot.

En d'autres termes, on peut tout à la fois être convaincu par les résultats de l'enquête et de l'analyse de l'auteur (davantage d'échanges entre pilotes et concepteurs en amont de l'innovation diminueraient cette cause potentielle d'accident qu'est le désarroi d'un pilote devant une machine qu'il ne comprend pas) et se souvenir que, lors des accidents -c'est-à-dire lors de ces « moments d'exception » auxquels elle a choisi par méthode de ne pas s'intéresser pour concentrer son enquête sur le «quotidien » (CM:69)-, les défaillances des pilotes (ou de la maintenance) l'emportent sans doute sur celles de la machine. Tout le livre de Moricot démontre qu'une partie de ces faiblesses résultent d'un mauvais dialogue des hommes avec des systèmes souvent mal adaptés, ou qui exigent des gens « de se comporter de manière inappropriée » (Norman 1993). Mais, à lire Flying, la faillibilité des pilotes est sans doute plus grande que ce qu'en pensent les équipages eux-mêmes. Tel est d'ailleurs le point de vue de ces acteurs essentiels du développement de nouveaux avions de ligne et des « systèmes » qu'ils embarquent que sont les pilotes d'essais auxquels est consacré le livre dirigé par V. Scardigli.

Résultant d'une recherche effectuée pour la Direction Générale de l'Aviation Civile, Comment naissent les avions est un ouvrage à plusieurs voix qui soulève à son tour la question des rapports entre les hommes et les automates dans le domaine 
aéronautique. Fondé sur des enquêtes menées entre 1996 et 1998 au Centre d'Essais en Vol d'Istres (CEV) -où des agents de l'État "certifient» les appareils et les équipements- et auprès des pilotes et ingénieurs d'essais des constructeurs Airbus Industrie et Aérospatiale à Toulouse, ce livre décrit lui aussi une période passée (les choses évoluent vite) mais cruciale, pendant laquelle se sont révélés le malaise d'une profession particulière et les tensions qui la traversent. Posant comme hypothèse que les pilotes d'essai constituent une collectivité humaine suffisamment restreinte et homogène pour partager "une histoire fondatrice, des valeurs et des rites, une explication du monde et un projet d'action » (VS : 32, note 7), ses auteurs se demandent de quoi et comment est constitué cette "culture » des essais, mais également quel est (ou pourrait être) son rôle dans la conception des avions et leur certification par la puissance publique. Mais qu'est-ce qu'un pilote d'essai?

Précis à souhait, le livre apporte plusieurs réponses à cette question. Jadis, du temps de ces «anciens» dont le souvenir hante l'École du Personnel Navigant d'Essais et de Réception (EPNER) -les Charles Goujon, Kostia Rozanoff, Jacqueline Auriol ou André Turcat qui se mêlaient à Buck Danny ou Dan Cooper dans les rêves des petits garçons des années 1950 et 1960-, un pilote d'essai risquait sa vie pour étudier les réactions générales d'un prototype, en évaluer les qualités de vol ou battre des records qui constituaient autant de points marqués pour l'industrie nationale. Il n'était pas rare que la mort soit au rendez-vous, et Scardigli peut raisonnablement parler de la fonction " sacrificielle» de ces pilotes qui mettaient leur vie en jeu pour que d'autres équipages, puis des milliers de passagers, voyagent dans une relative sécurité. Qu'ils fussent pilotes, ingénieurs ou mécaniciens, ceux qui effectuaient ces centaines de vols (il y eut plus de 300 prototypes évalués au CEV entre 1945 et 1960) étaient considérés comme les co-inventeurs des appareils. À mesure du développement de la conception assistée par ordinateur (on parle de "SAO ", "spécification assistée par ordinateur ») et du recours toujours plus grand aux simulateurs de vol, qui permettent aux pilotes de se familiariser avec les réactions probables de l'appareil avant son premier vol, les accidents ont beaucoup diminué. De nos jours, « les avions sont souvent dérivés les uns des autres" (Turcat 2000: 61), ce qui limite encore l'imprévu. Le risque n'a pas totalement disparu (accident de l'Airbus A-330 en 1996), mais le principal «sacrifice » attendu des pilotes d'essai est celui de leur vie privée ou d'un salaire qui pourrait être plus élevé.

Leur tâche même a changé : il ne s'agit plus de tester empiriquement, par essais et erreurs, l'aptitude générale d'un appareil à évoluer, ni même de vérifier les performances ou les qualités de vol annoncées par un constructeur comme dans les années 1970, mais de porter des jugements sur des "systèmes ", sur la " visualisation " offerte par des écrans, ou sur la manière dont un pilote de ligne utilisera l'avion'. Cependant, les pilotes ou les ingénieurs d'essai continuent de se singulariser par leur polyvalence (ils connaissent beaucoup d'avions) et par la détention d'un savoir et d'un savoir-faire spécifiques : celui qui leur permet d'explorer les limites du domaine de vol d'un avion, d'imaginer où peut se situer la «faille " d'un système, ou de se mettre volontairement à la place d'un pilote de ligne " moyen ». Bref, ils partagent une même "philosophie de l'essai», dont les auteurs montrent qu'elle s'acquiert lors de leur passage à l'EPNER.

Aujourd'hui située à Istres, à proximité du CEV, l'école forme en un an des navigants d'essai (des pilotes, ingénieurs ou mécaniciens d'avion ou d'hélicoptère, mais aussi des 
contrôleurs aériens) auxquels on apprend ce que doit être un essai en vol, c'est-à-dire à imaginer des questions pertinentes, à conduire une expérimentation, puis à «verbaliser leur intuition ». Simultanément, on cherche à faire acquérir aux stagiaires une «vision du monde qui rende compatibles celles des deux autres collectivités professionnelles" (les concepteurs et les pilotes de ligne; VS : 82). Ils découvrent notamment que leur travail consiste à parvenir à un compromis entre les exigences ou les besoins de ces acteurs; par exemple, à tenir compte des enjeux économiques colossaux d'une certification. On leur transmet là une " pensée de l'essai » qui résume les «valeurs » des navigants d'essai : douter systématiquement; dénicher ce qui peut mettre en jeu la sécurité d'un appareil confié à un pilote " moyen »; concilier les points de vue des autres acteurs de la communauté aéronautique.

Mais cet enseignement d'une "culture des essais », dont Scardigli décrit en détail les lieux et les processus d'acquisition (notamment des références constantes à l'histoire de l'École et du CEV) est «insuffisant pour rendre compte de la cohérence de la population concernée " (VS : 89). Les élèves réalisent également ce qui les différencie des «autres». Des pilotes de lignes, d'abord, qui s'apparentent (paraît-il) à des "singes ", ont du mal à comprendre qu'un système représente un compromis et n'imaginent pas que la passion amène un pilote d'essai à gagner moins d'argent qu'il n'en gagnerait ailleurs. Mais aussi de leurs confrères étrangers, car les essais «à la française » ont la particularité d'être effectués par un couple ingénieur-pilote, c'est-àdire de rassembler autour d'une même tâche des spécialistes venus de métiers différents, qui se trouvent forcés de parler un « langage commun » (VS : 102-103). Après cinq années passées au CEV, les navigants d'essai se dispersent. Parmi les pilotes, beaucoup retournent aux escadrilles de chasse dont ils sont issus ; quelques-uns (ils ont alors environ quarante ans) rejoignent la division «essais » d'un constructeur. Ainsi que le montrent longuement les auteurs, ces pilotes d'essai venus de l'organisme étatique de certification et passés dans le privé comptent pour beaucoup dans les relations informelles qui, en cas de divergence de vues sur une modification à apporter à un appareil, permettent aux experts du CEV et aux représentants d'un constructeur d'aboutir à un accord. En d'autres termes, par leur passage par l'EPNER puis au CEV et par la valeur qu'ils y accordent aux essais en vol, les anciens d'Istres constituent un élément de dialogue et d'entente crucial dans les relations conflictuelles entre les différents acteurs du monde aéronautique. Argument d'autant plus convaincant que tout le livre montre que, par leur métier même, les navigants d'essai sont des " passeurs ", toujours situés entre deux ou plusieurs de ces acteurs.

Et cela, d'abord entre les pilotes de lignes et les constructeurs, même si C. Moricot montre que ce rôle leur est contesté par les premiers. Selon les constructeurs, pour qui le Flight Management System doit se rapprocher de l'automate intégral, le pilote est -ou devrait être- un simple opérateur: la machine s'occupe du reste. Pour peu que la communication soit bonne entre le commandant de bord et le co-pilote et que ceux-ci utilisent correctement les "systèmes " installés dans les cockpits, notamment en se pliant aux consignes de Cockpit Ressource Management, la sécurité est assurée. Pour leur part, les pilotes s'insurgent contre ces ordinateurs qui peuvent devenir des «traîtres " (CM : 210). Sans cesse, le pilote d'essai et son double, l'ingénieur, doivent donc se mettre à la place d'un pilote "moyen »: à la place du pilote fatigué, voire fatigué et surpris par un incident ou une information donnée par le « système » de l'avion. Ils font alors volontairement des erreurs pour chercher celles qui mettraient l'avion en danger. Eux savent que les pilotes peuvent en faire, singulièrement avec une machine qui cache 
des informations ou avec une machine dont la logique est surprenante. Le pilote d'essai réintroduit la "pluralité des possibles et de l'imprévisibilité partielle du réel » (VS: 201). De l'existence de ces faiblesses de pilotes qui ne sont pas les opérateurs dociles dont rêvent les concepteurs, ils doivent convaincre les constructeurs. Outre une vision particulière des relations entre l'homme et la machine, ces derniers ont des arguments sans rapport avec la sécurité ou la facilité du pilotage : toute modification demandée par le CEV a un coût, qui peut être exorbitant.

Divers facteurs compliquent encore davantage ces réseaux d'« intermédiations » dont les auteurs exposent parfaitement les agents et les tensions. Par exemple, à l'échelle de l'entreprise qui les emploie, les pilotes d'essai des constructeurs ont, vis-à-vis des ingénieurs des bureaux d'étude, les mêmes revendications qu'ont les pilotes de ligne: ils se plaignent eux aussi de ne pas disposer de suffisamment d'informations sur les systèmes qu'on leur demande de tester. Quant aux concepteurs, ils reprochent à ces essayeurs «maison» d'être «allés voir dans les coins du domaine de vol» (VS : 154), c'est-à-dire de ne pas épouser la vision que le constructeur a des choses (sous-entendu, si le pilote se comporte en opérateur ordinaire, le système n'amènera pas l'avion à la limite de ses performances). Il arrive aussi que des considérations politiques ou économiques conduisent la hiérarchie du CEV à désavouer les conclusions de ses experts.

La place même des pilotes d'essai de l'État est aujourd'hui contestée. D'abord, du fait de l'internationalisation des procédures de certification et du développement de la simulation, les vols d'essai sont de plus en plus rares. De plus, la complexité et les interrelations des systèmes embarqués sont telles que « les scénarios de comportement de l'avion sont innombrables, donc impossibles à vérifier exhaustivement» (VS : 328). Bref, les navigants d'essai ne sont plus considérés comme infaillibles; en tout cas, ils ont perdu une partie de leur aura, ce qui affaiblit leur légitimité sociale. D'autre part, les pilotes des compagnies participent désormais à la conception des machines; les vols d'essais sont aujourd'hui plus nombreux chez les constructeurs qu'au CEV. Plus généralement, les constructeurs contestent aux experts publics le droit de s'enquérir de la logique des systèmes installés sur les avions, ce qui renforce encore le pouvoir des premiers dans le monde aéronautique. Et même lorsqu'il dispose des informations dont il a besoin, qui l'expert doit-il défendre, un constructeur national ou le public (VS: 336-337) ${ }^{10}$ ?

34 Les rapports entre les États, les industriels, les compagnies et les usagers, pilotes ou passagers, se trouvent aujourd'hui modifiés. Le choix d'un dispositif de guidage des avions, par exemple, ne détermine pas simplement un moyen de réguler le trafic aérien : c'est aussi une option industrielle à très long terme, lourde d'implications politiques, et qui concerne les citoyens autant que les professionnels de l'aéronautique. Comme l'indiquent deux chapitres du livre analysant les controverses entre constructeurs et certificateurs à propos d'un système de navigation embarqué, le pilote d'essai ou l'ingénieur de conception sont amenés à se prononcer sur des questions susceptibles de redéfinir les règles et les acteurs du contrôle aérien, alors même qu'ils « n'ont (pas) reçu mandat pour concevoir la société de demain » (VS : 320).

Les auteurs décrivent une situation inquiétante qui parait relever de l'illusion des patineurs (« il y a beaucoup de monde sur le lac gelé, donc la glace doit être solide») : dans la période actuelle, "chacun adopte une posture de confiance dans les autres acteurs. Le pilote partage la conduite de son destin avec un automate, dont l'action lui 
semble pourtant en partie imprévisible; le concepteur sait que le pilote est faillible, mais l'utilise comme dernier rempart contre l'accident; chacun se repose sur le certificateur, garant de la sécurité de systèmes informatiques que nul ne pourrait examiner exhaustivement» (VS: 24). Afin que de tels arbitrages éthiques et sociopolitiques soient démocratiquement rendus, les auteurs préconisent l'institution d'« un lieu neutre de réflexion et d'élaboration des choix aéronautiques » (VS : 230). Avec l'aide des sciences sociales, bien sûr, mais aussi, si j'ai bien compris, avec celle de navigants d'essai. L'homogénéité du groupe que constituent ceux-ci est certes toute relative (VS : 325), mais, jugés irremplaçables, de par leur « culture » de l'essai en vol et leur expertise, ils sont les mieux qualifiés, aussi bien pour contribuer à l'évolution des réglementations que pour assurer la sécurité de tous lors de la certification. Dans ce dispositif, l'EPNER aurait une place essentielle, à la fois par la «philosophie » qu'elle parvient à faire partager à tous les pilotes d'essai, mais aussi du fait que son credo, « les essais demeureront toujours nécessaires ", devrait s'imposer à tous "parce que le vol aux extrêmes reste un acte qualitativement différent de ce que peut apporter toute autre démarche » (VS : 107, 344, souligné par Scardigli).

Denses mais bien construits, et l'un et l'autre accessibles au non-spécialiste (même si le texte de Moricot est d'une lecture plus aisée que celui de Scardigli et de ses collaborateurs, où la multiplicité des voix est sensible), ces deux livres de sociologie des techniques atteignent leur but: donner de la dimension socio-technique de l'aéronautique une vision globale fondée sur des enquêtes de terrain qui permettent de voir et d'entendre parler ses acteurs. Ils s'inscrivent dans un projet à long terme ${ }^{11}$ particulièrement réussi, qui montre ce que les sciences humaines peuvent apporter à la compréhension des mécanismes et des enjeux multiples de phénomènes contemporains qui nous concernent tous, en tant qu'usagers ou citoyens. Il s'agit là de recherches appliquées, ou applicables, qui devraient convaincre les « décideurs » de l'utilité d'une telle approche. La chose est suffisamment rare pour être soulignée et me conduit à revenir un instant sur cette "socio-anthropologie» du monde contemporain qui mêle avec succès l'enquête de terrain et les questions de la sociologie de l'innovation. Pour le dire brièvement, certaines analyses de ces auteurs -ou, plutôt, certaines de leurs interprétations de détail- me semblent moins crédibles que d'autres et, donc, susceptibles d'affaiblir leurs démonstrations.

Quelques-unes de ces exégèses discutables sont secondaires. J'en citerai deux, cependant, qui pourraient ne pas emporter l'adhésion d'un membre de la communauté aéronautique. Premier exemple, lorsque, à propos des procédures de vérification du comportement d'un avion aux basses vitesses (et des risques de décrochage), l'instructeur de l'EPNER déclare: «Pour la certification de Concorde, c'est une voilure delta, il n'y a pas vraiment de Vs [vitesse de décrochage] » (VS : 56), le sociologue décèle dans ses paroles une "réappropriation du Concorde» (VS: 59), en tant qu'avion prestigieux, ou élément d'un «mythe fondateur » (VS : 60). C'est bien possible, mais on peut aussi, ou d'abord, considérer que l'instructeur ne veut pas dire autre chose que ce qu'il dit : une voilure delta ne décroche pas ${ }^{12}$. Il y a simplement là, de sa part, un rappel d'une réalité physique et technique. De même, là où l'instructeur explique que, lors d'essais de vol sur un moteur «sur un avion qui a 35 ans d'âge, on met un moteur au ralenti au lieu de l'arrêter », V. Scardigli parle du «supplément d'âme -ou de prudence 
- que l'on doit témoigner à une personne âgée : on ne poussera pas l'essai jusqu'au bout avec un très vieil avion" (VS: 59). J'imagine que devant cette interprétation psychologisante de ces paroles, l'instructeur pourrait tout aussi bien faire remarquer au sociologue qu'il ne s'agit pas d'essayer un très vieil avion, mais plutôt d'apprendre à faire un essai avec la vieille Caravelle du $\mathrm{CEV}^{13}$. Dans la phrase citée, il ne s'agit pas tant de respecter l'avion que les stagiaires, dont la vie serait en danger si une vraie panne de moteur affectait l'appareil sur lequel ils apprennent leur métier. Bref, on a là un indice supplémentaire de cette culture de la prudence que l'on pratique au CEV. Ces points paraîtront mineurs, mais ils sont susceptibles de faire douter un homme de l'art de l'analyse que lui propose le sociologue. Et, comme on l'a compris, rien ne serait plus regrettable.

Les allusions répétées au "rituel» et au "chamanisme» sont plus sérieusement dommageables. Commençons par les chamanes. Tout le livre coordonné par Scardigli démontre que, tant la spécificité que les difficultés de la profession de pilote d'essai sont liées aux positions d'intermédiaires qui sont les leurs: entre les pilotes des compagnies et les constructeurs; entre l'État qu'il représente au CEV et les intérêts privés que défendent leurs collègues payés par les constructeurs; entre la puissance publique, qui leur demande de réfléchir à de profondes mutations de la régulation aérienne et les citoyens. Ils sont des " passeurs de frontières ». Fort bien, mais pourquoi leur accoler l'un des concepts les plus flous de l'anthropologie, celui de "chamane »? On peut discuter sans fin l'utilité de cette catégorie (le chamanisme est-il un phénomène dont seule importe l'étude locale, dans un système religieux précis ? Ou bien, y a-t-il un intérêt à s'interroger sur les caractéristiques générales du phénomène?). Mais la plus concise des définitions du chamanisme parle d'un personnage dont une ou plusieurs composantes spirituelles voyagent dans l'invisible, au pays des morts, dans le monde des esprits, chez des maîtres du gibier, etc. Quel rapport existe-t-il alors entre un chamane et un automate (le "pilote automatique " qui se présente comme une "émanation des concepteurs, qui iraient rendre visite à l'équipage en vol »;VS : 237) ? La remarque sur l'inscription -délégation, dirait Latour - dans un dispositif technique d'une logique (voire de la vision du monde ou de l'éthique) qui n'est pas celle des pilotes et s'impose à eux est capitale. Mais à quoi bon ajouter "Cela évoque l'esprit du chamane... »? En vérité, on espère sincèrement que l'ambigu respect que les pilotes de ligne portent aux pilotes d'essai s'explique sans que les premiers confondent les seconds avec des chamanes. Mais, surtout en quoi ce rapprochement sert-il la démarche du sociologue? À mes yeux, il ne peut que la desservir.

Passons à une autre "métaphore» (comme dit C. Moricot), celle des « rituels». Autant que le lieu d'un "rite d'institution » où les élèves sont conviés à porter le poids d'une histoire qui les dépasse (Bourdieu), l'EPNER serait un dispositif initiatique. Pourtant, si l'on peut admettre avec les auteurs qu'en se concentrant pendant un an sur leur formation, des hommes (en majorité) d'une trentaine d'années subissent une « mise à l'écart » qui n'est pas sans rappeler celle qui ponctue un rite de passage, on doute qu'elle soit un endroit où «la fatigue fait tomber les défenses personnelles, facilitant l'adhésion aux systèmes de valeurs et à la représentation du monde présentée par les leaders du groupe » (CM : 47, 75, 85, 89). Si les élèves pilotes d'essais volaient dans un état d'abrutissement rappelant moindrement celui des initiés anga que je côtoie, mieux vaudrait immédiatement évacuer la bonne ville d'Istres et ses environs ! 

cette fois, aux pratiques des équipages au cours de ces « rotations » qu'elle a été la première sociologue à pouvoir observer de bout en bout dans un cockpit. Rien ne s'oppose à l'emploi de termes comme « rite » ou « rituel » pour désigner ce mélange de répétition de gestes et de paroles codifiés que l'on y observe -check-list, " annonces » d'informations relatives à des tâches "stratégiques" d'un pilote à l'autre- dont l'aspect est parfois «solennel» (ou nous paraît solennel). On peut de même suivre l'auteur lorsqu'elle suppose que ces répétitions de gestes et de paroles ont une « efficacité qui ne s'épuise pas dans l'instrumentalité technique du lien cause-effet »; ou lorsqu'elle précise que cette efficacité de la « répétition codifiée [...] s'exprime aussi dans l'adhésion à un système de valeur qui est celui de la sécurité et surtout dans la croyance en la légitimité de ce système de valeurs " (CM : 280-281). Tout dépend de ce que l'on met dans le terme « croyance». Pour un ethnologue spécialiste des rituels, la répétition de listes est assurément l'une des dimensions possibles d'un rituel, qui joue un rôle central dans la mémorisation (et la transmission entre les générations) d'une pratique religieuse. Et il ne fait pas de doute que réciter une check-list n'a pas pour seul « effet » de vérifier l'état des systèmes de l'avion dans lequel on se trouve : c'est aussi communiquer dans un langage commun avec un copilote inconnu; un moyen de se souvenir le lendemain de chaque point de la procédure; et, pourquoi pas (c'est indémontrable), un rappel de plus que la sécurité est une « sacrée » question ( $\mathrm{CM}: 315)$. On en dirait autant de la visite pré-vol : chercher des traces de fuites, inspecter des aubes d'une soufflante de réacteur d'un rapide regard ou secouer l'extrémité d'une hélice (ou d'une aile) peut paraître totalement inutile (ou « rituel »). À ce détail près qu'il arrive qu'un avion perde une aile en vol, du moins en Papouasie Nouvelle-Guinée, et qu'il est probable qu'une inspection au sol peut réduire ce type de risque fâcheux.

On m'objectera que Humphreys \& Laidlaw (1994) citent précisément parmi les exemples de comportement ritualisé celui d'un pilote venu de la RAF qui appliquait à son Jumbo Jet la visite pré-vol largement inutile qu'il avait utilisée toute sa vie pour des avions de chasse -en faisant le tour d'une dérive qu'il n'est plus question de secouer de la main car elle est inaccessible du sol, par exemple. Mais, c'est précisément parce que l'inspection de ce pilote n'a plus d'efficacité matérielle, parce qu'elle s'applique à un objet dont l'examen relève d'un autre corps de métier et échappe désormais à sa compétence (Humphreys \& Laidlaw 1994: 158), parce qu'elle est "non technique" (Whitehouse $2002: 46$ ), que ces spécialistes y voient un rituel ${ }^{14}$. Or, qui prétendrait que la check-list égrenée dans un cockpit d'Airbus n'a pas d'efficacité technique ? Jusqu'à nouvel ordre -c'est-à-dire jusqu'à ce que le détail des procédures gestuelles et verbales des pilotes ait été comparé avec ce que l'on sait des gestes et paroles rituels dans un cadre religieux-, une check-list est un moyen de s'assurer rapidement, y compris avec un copilote avec lequel on vole pour la première fois, que tout est en ordre. En rater une étape peut être dramatique : j'ai le souvenir (mais pas la référence précise, si ce n'est qu'il s'agissait là encore d'un article de Flying) de cet accident causé par un pilote qui, peu avant d'atterrir, avait entrecoupé sa check-list de considérations sur le temps qu'il faisait «en bas» et sur l'agréable partie de tennis qui l'attendait. Oubliant de vérifier le bon fonctionnement des pompes hydrauliques, il avait posé (en fait écrasé) son appareil à une vitesse supérieure de $100 \mathrm{~km} / \mathrm{h}$ (était-ce 100 nœuds ?) à la vitesse requise, faute d'action des volets hypersustentateurs. Ceci montre au moins que tous les pilotes ne reconnaissent pas la même valeur aux check-lists. Ou que certains les récitent « sans y croire ", serait-on tenté d'écrire. De plus, comme le fait fort justement 
remarquer C. Moricot, pour que ces gestes et paroles constituent un rituel, il faudrait "y ajouter la dimension sacrée et symbolique sans laquelle la "cérémonie" n'aurait pas lieu d'être " (CM : 281). Convoquer les «symboles ascensionnels » de G. Durand (1984) pour ajouter du sens ou du sacré aux check-lists ne me paraît pas s'imposer ; pas plus que le rapprochement formel avec la prière ( $\mathrm{CM}: 308)$.

En résumé, la qualité des enquêtes et des arguments de Moricot ou de Scardigli est telle qu'elle devrait amplement suffire à convaincre les décideurs de confier aux spécialistes des sciences humaines davantage d'études des techniques modernes. Leurs ouvrages illustrent magnifiquement l'application potentielle des travaux de sociologie mâtinés de technologie culturelle à la prise de décisions techniques et politiques dans la société industrielle contemporaine. Mais les références au rituel, à l'altérité et autres chamanismes n'apportent rien à leurs arguments.

Outre le risque qu'elles font courir à la crédibilité de ces remarquables travaux de sociologie des techniques modernes, ces analogies discutables avec des situations ethnographiques "exotiques » soulèvent donc les vieilles questions de l'efficacité symbolique ou de la frontière entre "technique " et "social». Comme on le sait (Akrich 1994, Latour 1994), toute la difficulté consiste à dissoudre cette frontière qui n'existe que dans nos têtes, même s'il peut être pratique de la maintenir dans la description et l'analyse (Lemonnier 1994). Qu'il s'agisse de poteries ou d'aéroplanes, le dilemme reste le même, avec cette difficulté supplémentaire qu'introduire un spécialiste de sciences humaines dans un cockpit (un atelier, une tour de contrôle...) pose également la question de l'application de concepts classiques de l'ethnologie à l'étude du monde industriel contemporain.

Quoi qu'il en soit, l'une des réussites des travaux de Moricot et Scardigli est de donner de techniques de pointe de l'aéronautique une image qui, pour être complexe, ne déroute en rien un ethnologue spécialiste d'objets infiniment moins modernes qu'un aéroplane. Au point que l'on serait tenté de compléter l'approche des sociologues (celle qui parle de "pouvoir", de "légitimité » ou de "controverses») par une prise en compte de ces questions de base de l'ethnologie des techniques, qui concernent la logique des variantes et des choix, ainsi que les représentations que les acteurs se font de ces variantes et de ces choix. Les experts du CEV nous mettent sur la voie, qui construisent leur "altérité» en expliquant combien leurs méthodes et leur "philosophie de l'essai » diffèrent de celles des navigants d'essai américains (VS: 101-104). La fréquentation de pilotes de brousse en Papouasie Nouvelle-Guinée ou la lecture de Flying indique que les représentations de la sécurité et des procédures susceptibles de l'augmenter sont loin d'être homogènes. Varient-elles selon les pays ? Selon les catégories d'avions pilotés par les professionnels (masse ou nombre de passagers)? Selon la carrière des pilotes (et, notamment, leur éventuel passé de militaire) ? Le champ des variantes ou des choix individuels et collectifs est immense.

Qu'il s'agisse de comparer les représentations de la sécurité chez un jeune Australien venu accumuler des heures de vol en Papouasie ou chez un pilote missionnaire ayant Dieu comme copilote, de cerner en quoi diffèrent les pilotes d'aviation générale qui lisent Flying des pilotes professionnels qui écrivent la revue, ou de tout autre thème de recherche ayant le pilotage des avions en filigrane, les travaux pionniers de Moricot et Scardigli constitueront ici une référence. Mais on est prié de laisser son chamane au vestiaire. 


\section{BIBLIOGRAPHIE}

Akrich, Madeleine

1994. « Comment sortir de la dichotomie technique/société. Présentation de diverses sociologies de la technique », pp. 105-131, in B. Latour, P. Lemonnier (eds), De la préhistoire aux missiles balistiques. L'intelligence sociale des techniques. Paris : La Découverte.

Bénichou, Michel

2000. Un siècle d'aviation française. Clichy : Docavia, Éditions Larivière.

Callon, Michel

1986. «Éléments pour une sociologie de la traduction. La domestication des coquilles SaintJacques et des marins-pêcheurs dans la baie de Saint-Brieuc ", L'Année sociologique 36 : 169-208.

Bodemer, Alfred \& Robert Laugier

1979. Les moteurs à piston aéronautique français (1900/1960). Paris : Docavia.

Crouch, Tom D., Jakab, Peter L.

1994. « Les frères Wright », Icare, revue française de l'aviation 147 : 10-105.

Chadeau, Emannuel

1987. L'industrie aéronautique en France. 1900-1950. Paris : Fayard.

1991. « Voisin contre Wright ou l'analyse d'un duel », Les Cahiers de Science \& Vie 1:38-57.

Dudal, Pierre

1998. « Un pilote d'Air France aux essais en vol », Icare. Revue de l'aviation française 164 : 148-157.

Durand, Gilbert

1984. Les structures anthropologiques de l'imaginaire. Paris : Dunod.

Edgerton, David

1991. England and the Aeroplane. An Essay on a Militant and Technological Nation. London : MacMillan.

Gaillard, Pierre

1994. « Le cinquantenaire du Centre d'Essais en Vol », Le Fana de l'Aviation 300 : 50-58.

Garrison, Peter

1985. « Aftermath. Bounced Checks », Flying 112 (4) : 18-19;

1994. « Aftermath. Cast the first stone », Flying 121 (12) : 92-94.

1998. « Aftermath. Interface », Flying 125 (5) : 101-103.

1999a. " Aftermath. Misloaded, mistrimmed », Flying 126 (1) : 87-88.

1999b. « Aftermath. Emergency descent », Flying 126 (2) : 87-90.

2003. «Aftermath. Heads in the Clouds », Flying 130 (7) : 90-91

Hooven, Frederick J.

1978. « The Wright Brothers Flight-Control System », Scientific American 239 (5) : 167-184.

Humphreys, Caroline, Laidlaw, James

1994. The Archetypal Actions of Ritual : a Theory of Ritual illustrated by the Jain Rite of Worship. Oxford : Oxford University Press.

Hurel, Jean-Marie

1995. Entre ciel et mer. Le commandant Maurice Hurel. s.l. : Éditions Imagine. 
Latour, Bruno

1992. Aramis, ou l'amour des techniques. Paris : La Découverte.

1994. La clef de Berlin et autres leçons d'un amateur des sciences. Paris : La Découverte.

Lemonnier, Pierre

1994. «Choix techniques et représentations de l'enfermement chez les Anga de Nouvelle-

Guinée ", pp. 253-270, in B. Latour et P. Lemonnier (eds), De la préhistoire aux missiles balistiques.

L'intelligence sociale des techniques. Paris : La Découverte

1996. «Et pourtant ça vole! L'ethnologie des techniques et les objets industriels », Ethnologie

française 26 (1) : 17-31.

Lemonnier, Pierre (ed.)

1993. Technological Choices. Transformation in Material Cultures since the Neolithic. London : Routledge (réédition en paperback, 2001)

Mackenzie, David

1991. Inventing Accuracy. A Historical Sociology of Nuclear Missile Guidance (Inside Technology).

Cambridge (Mass.) : The MIT Press.

Nahum, Andrew

1987. The Rotary Aero Engine. Londres : The Stationary Office (Science Museum).

Norman, Don A.

1993. Things that make Use Smart. Defending human attributes. Readin (MA) : Addison Wesley

Publishers.

Owen, Kenneth

1982. Concorde. New shape in the sky. London : Jane's Publishing Company, in co-operation with the Science Museum.

Phelan, P.

1996. «Stormy passages. For a nation dependent on air transport, Papua New Guinea has many problems to overcome », Flight International $10: 38-39$.

Quilici-Pacaud, Jean-François

1993. «Dominant representations and technological choices. A method of analysis with examples from aeronautics ", pp. 399-412, in P. Lemonnier (ed), Technological Choices. Transformation in material culture since the Neolithic. London : Routledge.

2000. «Cent ans d'ailes et de pneus ", Analyse de systèmes 26 (1-2) : 5-57.

Sparaco, Pierre

2002. Concorde. La véritable histoire. Clichy : Docavia, Édition Larivière.

Turcat, André

2000. Concorde. Essais d'hier, batailles d'aujourd'hui. Paris : Le Cherche-midi éditeur.

Vincenti, Walter G.

1990. What Engineers Know and How They Know It : Analytical Studies from Aeronautical History. Baltimore (MD) : The John Hopkins University Press.

1994. "The retractable airplane landing gear and the Northrop "Anomaly" ", Technology and Culture 35 (1) : 1-33.

Whitehouse, Harvey

2002. "Conjectures, refutations, and verification : towards a testable theory of "modes of religiosity" ", Journal of Ritual Studies 16 (2) : 44-59. 


\section{NOTES}

1. Mais j'ai encore pillé le savoir de Jean-François Quilici-Pacaud, pilote militaire et ingénieur mécanicien, pour contrôler mes impressions d'amateur en aéronautique. Qu'il en soit à nouveau remercié.

2. J.-Cl. Fayer, Vols d'essais. Le Centre d'essais en vol de 1945 à 1960, Boulogne-Billancourt, E.T.A.I., 2001 (ci-après « Fayer I ») ; J.-Cl. Fayer, Prototypes de l'aviation françasie, 1945-1960. Boulogne-Billancourt, E.T.A.I., 2002 (ci-après « Fayer II »).

3. Le splendide ouvrage de MacKenzie $(1991,1994)$ sur le guidage des missiles atomiques américains de la guerre froide constitue une exception à ce désintérêt des sciences humaines pour l'histoire des techniques aéronautiques.

4. Le cas de l'Aramis étudié par Latour (1992) reste ici un modèle.

5. C. Moricot, Des avions et des hommes. Socio-anthropologie des pilotes de lignes face à l'automatisation des avions (désormais « $\mathrm{CM} »$ ). Villeneuve d'Ascq, Presses universitaires du Septentrion, 1999. V. Scardigli, M. Maestrutti, J.-F. Poltorak, Comment naissent les avions. Ethnographie des pilotes d'essais. Paris, L'Harmattan, 2000 (désormais «VS »).

6.. Le site internet du fabricant d'un des plus récents systèmes à infrarouge propose des vidéos d'atterrissages effectués dans le noir absolu (www.max-viz.com).

7.. «L'erreur humaine », La Recherche, 1992, $216: 1444-1445$.

8. Dans le livre dont je parle un peu plus bas, Scardigli $(2000: 336)$ évoque le risque d'assister un jour à un relâchement des exigences de sécurité concernant des « "avions du pauvre", destinés aux compagnies charter ou au Tiers Monde ». L'article de Phelan (1996) donne des défaillances de l'Aviation Civile Papoue une image qui fait froid dans le dos ; mais il n'y est pas question des pilotes...

9. Comme le rappelait Gaillard (1994: 53), le CEMA d'avant-guerre comportait déjà une « Section Navigation-Pilote automatique-Équipements ».

10. La question de l'indépendance des pilotes d'essais n'est pas nouvelle : en 1923, le commandant Hurel hésitait à entrer chez le constructeur d'hydravions CAMS parce qu'il était un pilier de la Commission d'Études Pratiques d'Aéronautique, l'un des ancêtres du CEV (Hurel 1995 : 19).

11. Plusieurs travaux de la même équipe abordent d'autres aspects du monde aéronautique français, par exemple la thèse de $\mathrm{S}$. Poirot-Delpech sur l'automatisation du contrôle aérien (Biographie du CAUTRA [Contrôle Automatique du Trafic Aérien] Paris I, 1995) ou de V. Scardigli sur les systèmes d'aide à la navigation de l'Airbus A-320 (Un anthropologue chez les automates, Paris, PUF, 2001).

12. Comme l'explique P. Dudal, l'un des pilotes d'essai du supersonique, « une aile delta, c'est le cas de Concorde, ne décroche pas : plus l'incidence est grande, plus la trainée augmente et l'avion ne peut plus maintenir sa trajectoire : il s'enfonce, mais aucun phénomène ne vient perturber ses qualités de vol telles que vibrations ou abattée, du moins dans le domaine utile [...]. En d'autres termes, là où un avion classique ne vole plus, un appareil à aile delta peut encore évoluer» (Dudal 1998 : 151).

13. L'ouvrage de Fayer (2001: 379) parle d'une Caravelle essayée en 1956 et « qui sera ensuite utilisée de nombreuses années par le CEV »; peut-être s'agit-il de celle-là.

14. Cinquante ans de technologie culturelle ont démontré que les actions sur la matière comportaient des aspects sans "signification technique », sans effet sur la réussite de l'action sur la matière en cours de réalisation (au hasard, Lemonnier (ed) 1993). Je laisse de côté les déchirantes révisions que les spécialistes des rituels pourraient être amenés à apporter à la définition de leur objet de recherche s'ils lisaient Techniques \& culture. 
Cette question mériterait un autre article. Je remercie Harvey Whitehouse de m'avoir signalé l'exemple du pilote qui effectuait machinalement un « walk around » inutile.

\section{AUTEUR}

\section{PIERRE LEMONNIER}

Centre de Recherche et de Documentation sur l'Océanie, CNRS, Marseille 